\title{
rolyPOLY: A Hybrid Prototype For Digital Craft
}

\section{ANDREW JOHN WIT}

Temple University

The recent introduction of carbon fiber reinforced polymer (CFRP) based simulation tools and robotic fabrication has opened potentials in not only design, but also in full-scale testing in architectural applications. CFRP's unique properties such as high strength to weight ratio, malleability during fabrication as well as the capacity to create complex yet precise forms while minimizing waste through reconfigurable formworks allow for a high-level of precision and adaptability in architectural applications that traditional construction methodologies cannot afford.

Current methodologies of CFRP placement within industrial processes puts emphasis not on the visual, tactile and craft based potentials inherent within the material, but rather solely on producing optimized and repeatable systems. Unlike industrial fields where products are mass-produced to have identical dimensions, structural properties, and appearance; architecture requires the freedom and flexibility to freely alter configurations on a project-by-project basis. The creation of robotic-craft driven architectural artifacts, however, is complicated by the inherent complexity within these new tools and processes. For this reason this project reevaluates current methodologies of CFRP placement, investigating the benefits native to handcraft in relation to those of numerically controlled machines while being tested and evaluated through the completed artifact's optical, structural, functional and tactile merits.

\section{SIMON KIM}

University of Pennsylvania

This project board documents these questions though the recently completed wound CFRP prototype rolyPOLY. Completed in the winter of 2015 by means of an interdisciplinary collaboration between Andrew John Wit of Temple University and Simon Kim of the University of Pennsylvania, this completed project shows the exigencies and affordances between the realms of digital and analog. Through the integration of handcraft into the digital workflow, the project has reintroduced the potential for methods of rapid yet backwards-compatible revisions to winding rules, on-the-fly improvisation and intuitive alterations that previously would have been difficult to achieve during the robotic CFRP winding process. On the other hand, aspects inherent through digital and robotic production such as strength through speed, accuracy and repeatability can be simply reintroduced within subsequent projects. Through the winding process a more robust hybrid fabrication system, with a continuous dialog between human and machine, can be initiated. For the work documented here, rolyPOLY acts as a bespoke object in which to study ephemerality and perceptual effects such as texture, pattern interference, and variable levels of opacity or inherent patterning such as moiré. The discoveries gained through the prototype by human craftsmanship are then calibrated and formulated into rapid fabrication methodologies via machine processes.
This project board visually showcases the processes, outcomes as well as the potential of harnessing CFRP in craft based monocoque shell structures in architectural installations while maintaining high levels of structural rigidity and winding repeatability, as seen here in the rolyPOLY prototype. 

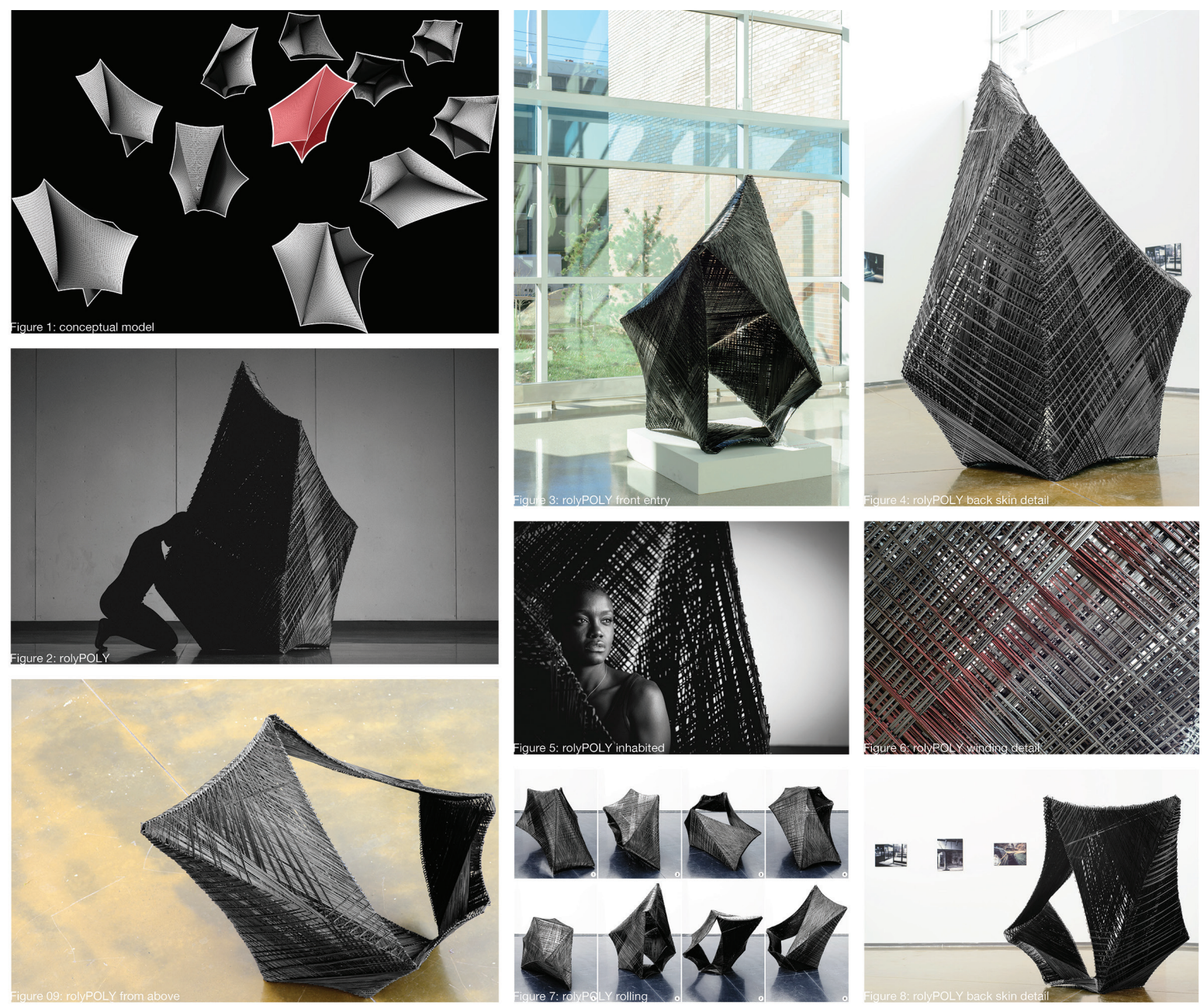

\section{rolyPOLY:}

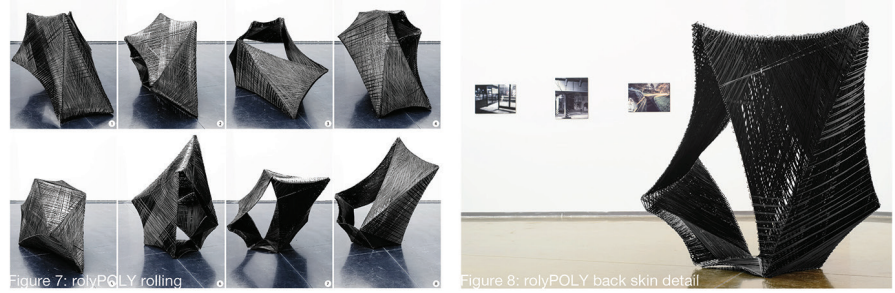

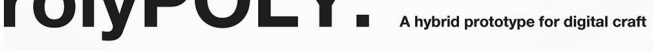
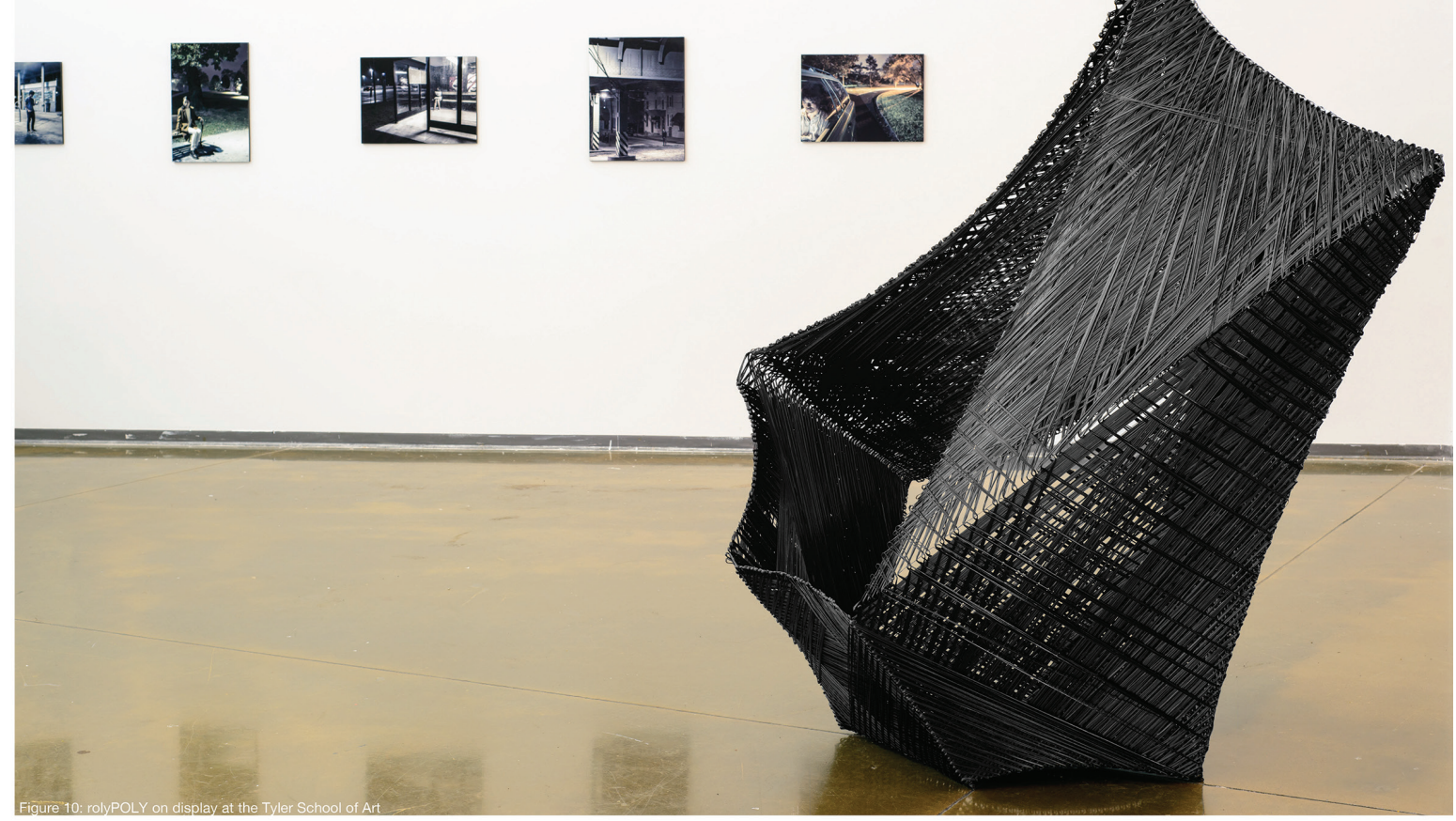